\title{
ADAPTACIÓN TRANSCULTURAL DE MENSAJES VÍA TELÉFONOS MÓVILES COMO APOYO PARA DEJAR DE FUMAR
}

\author{
CROSS-CULTURAL ADAPTATION OF TEXT \\ MESSAGES AS A SUPPORT TOOL TO QUIT \\ SMOKING
}

\author{
ADAPTAÇ̃̃O TRANSCULTURAL DE MENSA- \\ GENS VIA CELULAR PARA APOIAR A CESSA- \\ ÇÃO DO TABAGISMO
}

\section{Resumen}

Objetivo: Realizar la adaptación transcultural de SmokefreeTXT, intervención que envía mensajes de

María C. Ortiz León, ${ }^{1}$ James Thrasher, ${ }^{2}$ Jaime Morales Romero, ${ }^{3}$ Xóchitl De San Jorge Cárdenas. ${ }^{4}$

'Doctorado en Ciencias de la Salud. Universidad Veracruzana, México. ${ }^{2}$ University of South Carolina e Instituto Nacional de Salud Pública, Cuernavaca, México. ${ }^{3}$ Instituto Nacional de

Salud Pública. Universidad Veracruzana, México ${ }^{4}$ Instituto Nacional de

Salud Pública. Universidad Veracruzana, México

Trabajo recibido: 27 de Abril de 2017. Aprobado: 14 de Junio de 2017. texto para dejar de fumar en población hispana que vive en Estados Unidos de América al contexto cultural y lingüístico de México. Métodos:Utilizando un abordaje cualitativo, se efectuó la adaptación transcultural considerando aspectos teóricos, culturales y gramaticales para lo cual se realizaron grupos focales y entrevistas semiestructuradas en las que participaron fumadores y profesionales de la salud. La información recopilada permitió crear dominios y categorías, a partir de los cuales se integró la biblioteca adaptada de mensajes de texto. Resultados: Este proceso permitió identificar tres dominios, modificar 96 mensajes y crear nueve. La mayor parte de los cambios se realizaron en el dominio lingüístico y la biblioteca de mensajes quedó integrada por 182 mensajes de texto.Conclusiones: La intervención SmokefreeTXTse enriqueció con la precisión y generación de nuevos mensajes.

Palabras Clave: tabaco, mensaje de texto, teléfonos celulares, México. 
Objective: To carry out the cross-cultural adaptation of SmokefreeTXT, application used to send quit- smoking texts messages to the Hispanic population living in the United States, to the linguistic and cultural context of Mexico. Methods: The cross-cultural adaptation was carried out using a qualitative approach, considering theoretical, cultural and grammatical aspects. Focal groups were made and semi-structured interviews were applied to smokers and health professionals. The data collected allowed us to create domains and categories from which an adapted library of messages was integrated. Results: This process made it possible to identify three domains, modify 96 messages and create 9 . Most changes were performed in the linguistic domain; the library has a total of 182 text messages. Conclusions: The intervention SmokefreeTXTwas enhanced by making messages more accurate and generating new ones.

Key Words: tobacco, text messages, cell phones, Mexico.

\section{Resumo}

Objetivo: Realizar a adaptação transcultural do SmokefreeTXT, uma intervenção que envia mensagens de texto para parar de fumar em uma população hispânica que vive nos Estados Unidos da América para o contexto cultural e linguístico do México. Métodos: utilizando uma abordagem qualitativa, a adaptação transcultural foi realizada considerando aspectos teóricos, culturais e gramaticais, para os quais foram analisados grupos focais e entrevistas semi-estruturadas em que participaram fumantes e profissionais de saúde. As informações coletadas permitiram a criação de domínios e categorias, a partir das quais a biblioteca adaptada de mensagens de texto foi integrada. Resultados: este processo permitiu identificar três domínios, modificar 96 mensagens e criar nove. A maioria das mudanças foi feita no domínio linguístico e a biblioteca de mensagens ficou composta de 182 mensagens de texto. Conclusões: a intervenção SmokefreeTXT foi enriquecida com a precisão e geração de novas mensagens.

Palavras chave: Tabaco, Mensagens de texto, Celulares, México.

\section{Introducción}

La Organización Mundial de la Salud (OMS) estima que hay más de mil millones de fumadores en el mundo, de los que el $12 \%$ vive en las Américas (1), provocando aproximadamente 6 millones de muertes anuales, de las cuales 60000 ocurren en México (2). En respuesta, el artículo 14 del Convenio Marco para el Control del Tabaco (CMCT) propone medidas para reducir la demanda y la dependencia de tabaco y estrategias, farmacológicas y comportamentales, que deben basarse en la mejor evidencia disponible de su eficacia, ser accesibles, asequibles e incluyentes (3).

En la última década, se han desarrollado intervenciones comportamentales que se inscriben en el campo de la m-Salud y más específicamente de la m-Cesación, en particular los programas de envió de mensajes de texto cortos (SMS, por sus siglas en inglés) se han convertido en una de las herramientas más populares de la m-Cesación, ya que con sólo contar con un teléfono móvil cualquier fumador puede recibir apoyo para dejar de fumar(4).

Aunque la eficacia de este tipo de intervenciones sólo ha sido probada en población de países con ingresos altos en los que fueron diseñadas $(4,5)$,cumplen con las características establecidas en el artículo 14 del CMCT $(3,6,7)$. Se trata de una estrategia con enorme

\footnotetext{
${ }^{1}$ Uso de tecnologías de información y comunicación (TIC) móvil en la atención médica y la salud pública (33).
}

${ }^{2}$ Servicios que apoyan a los usuarios a dejar de fumar a través de dispositivos móviles. (13). 
potencial para la Salud Pública, que promete ser una opción costo-efectiva comparada con otros tipos de tratamiento (6), especialmente en países de bajos y medianos ingresos en los que los recursos para la deshabituación tabáquica son escasos (8)pero en los que se observa una tendencia creciente en el número de usuarios de teléfonos móviles (9).

La primera intervención de este tipo probada a nivel poblacional fue STOMP, desarrollada en Nueva Zelanda (10) y adaptada transculturalmente a Argentina (11) y a Reino Unido (12), en este último ya se ofrece como servicio a población abierta. En Costa Rica (13), único país de Latinoamérica que cuenta con este servicio ofrecido a través de su Ministerio de Salud, no encontramos evidencias sobre el proceso de adaptación ni de la evaluación de resultados. Actualmente se encuentra en revisión su plataforma computacional.

El Instituto Nacional de Cáncer de los Estados Unidos, ha sido pionero en la aplicación de las Tecnologías de Información y Comunicación (TIC) para apoyar la deshabituación tabáquica (14), por lo que puso a disposición de la población de habla inglesa en 2011 y de la población hispanoparlante en Estados Unidos en 2013, el servicio SmokefreeTXT, que envía SMS a teléfonos móviles, diseñados para motivar y apoyar a los fumadores que desean dejar de serlo (15). Ese mismo año, realizó una evaluación preliminar del servicio considerando datos de 13,145 usuarios, con tasas de abandono del consumo de tabaco de $11.3 \%, 9.8 \%$ y $9.5 \%$ al mes, a los tres y seis meses, respectivamente (16).Al comparar estas tasas con otro tipo de tratamientos, se encontró que se trata de una herramienta costoefectiva para apoyar a los usuarios que quieren dejar de fumar (17),razón por la que fue seleccionada para realizar la adaptación transcultural que presentamos.

\section{Adaptación transcultural de mensajes para dejar de fumar}

El CMCT establece la necesidad de la cooperación internacional para fortalecer el logro de sus metas (3); en este contexto, una de las líneas actuales de investigación en el campo de las intervenciones basadas en tecnología móvil para dejar de fumar, se refiere a la adaptación de aquellas que han demostrado ser eficaces en el entorno en donde fueron desarrolladas, a otros, con condiciones culturales y de desarrollo diferentes (18). Se trata de una adaptación transcultural (19),porque incluye todos aquellos elementos que la hacen compatible con los modelos culturales, significados y valores de la población destino, y en donde se cuida la equivalencia lingüística y conceptual así como la conservación de las propiedades de medida de la intervención original (20).

Para conocer las propiedades de cualquier intervención comportamental, es necesario identificar las técnicas de cambio conductual (BCT, por sus siglas en inglés), definidas como el componente activo que permite el cambio de comportamiento y que se caracteriza por ser observable, replicable e irreducible (21). Es importante identificar las BCT ya que, durante su desarrollo, SmokefreeTXT ha sufrido modificaciones y ha utilizado diversas teorías de cambio de comportamiento que le dan sustento teórico (E. Augustson, comunicación personal 8 de agosto 2015), como el modelo de las Etapas de Cambio o Teoría Transteórica (22),la Teoría Social Cognitiva (23), la Teoría de la Acción Planificada (24) y la Teoría de la Motivación (PRIME) (25). Algunas han mostrado ser más adecuadas en el desarrollo de un aspecto en particular; sin embargo, todas han aportado elementos comunes (26).

En la figura $\mathrm{N}^{\circ} 1$ se muestra el funcionamiento del servicio SmokefreeTXT diseñado para población hispanoparlante de Estados Unidos, resultado de una traducción literal del inglés, que no consideró las variantes regionales, culturales y lingüísticas de los distintos grupos étnicos entre los que se encuentran los migrantes mexicanos y que no ha sido evaluada para conocer si fue aceptada y entendida por ellos. 


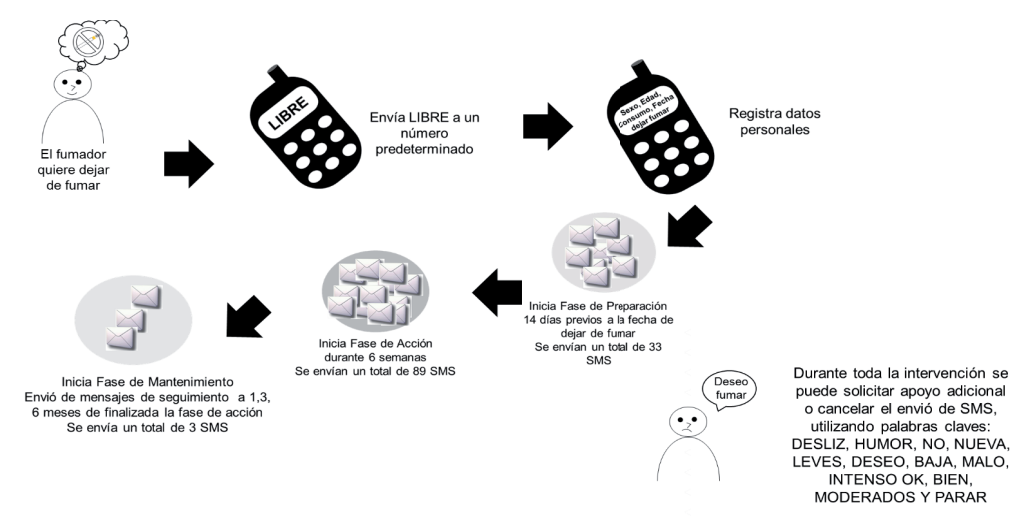

Figura $\mathrm{N}^{\circ} 1$ : Funcionamiento y estructura de SmokefreeTXT en español

En México, no existe ningún servicio institucionalizado de deshabituación tabáquica basado en él envió de SMS a teléfonos móviles que apoye a fumadores mexicanos que desean dejar de fumar. Por lo anterior, el objetivo de este estudio fue realizarla adaptación transcultural de la intervención SmokeFreeTXT utilizada en población hispana que vive en los Estados Unidos, al contexto cultural y lingüístico de México.Esta adaptación no sólo deberá tomar en cuenta la lengua, la redacción y el uso de palabras o expresiones familiares para ellos, sino también otros aspectos culturales como el tratamiento interpersonal entre otros, lo que justifica la adaptación transcultural de SmokefreeTXT para el contexto mexicano y en especial para el estado de Veracruz.

\section{Materiales y Métodos}

Desde una perspectiva cualitativa, se utilizó la metodología de laboratorios cognitivos sustentada en la propuesta de Barrera y colaboradores (19) que considera que la adaptación transcultural requiere de un proceso interactivo y gradual, que pasa por diferentes fases.

\section{Participantes}

Participaron tanto fumadores como personal de salud. En relación con los fumadores se utilizó un muestreo de máxima variación considerando sólo a mayores de edad beneficiarios potenciales del servicio. En total fueron 32 personas mayores de 18 años, 30 de los cuales eran fumadores activos y 2 en proceso de dejar de fumar, de ambos sexos, propietarios de un teléfono móvil, reclutados a través de Facebook, anuncios colocados en diferentes lugares de la ciudad de Xalapa, Ver. y utilizando la estrategia de bola de nieve. También invitamos a 10 expertos responsables del tratamiento contra el tabaquismo y seis profesionales de la salud de diversas especialidades, que tratan enfermedades atribuibles al consumo de tabaco.

\section{Procedimientos}

Antes de realizar la adaptación transcultural se corrigió la redacción de cada SMS para hacerlos comprensibles para los hablantes del español que se usa en México y particularmente en Veracruz. Posteriormente, participaron tres expertos en el campo de las adicciones quienes de manera individual identificaron las BCT, presentes yausentes enlos SMS, de acuerdo con la taxonomía de Michie y colaboradores (27). Para analizar los SMS, conformamos dos grupos focales de fumadores: el primero,

${ }^{3}$ Los mensajes que resultaron claros y comprensibles para todos los participantes recibieron la calificación de "aceptable" y se incluyeron en la intervención adaptada; en contraste, los mensajes que no cumplieron con estos requisitos o generaron dudas, fueron enviados a la revisión de los profesionales de la salud. 
integrado por 10 hombres jóvenes, en proceso de rehabilitación por el consumo abusivo de substancias ilegales; y el segundo, por 10 estudiantes universitarios $(50 \%$ hombres y $50 \%$ mujeres) del área de Ciencias de la Salud de una universidad pública. También se aplicaron entrevistas semiestructuradas a 12 fumadores activos o en proceso de dejar de fumar $(50 \%$ hombres y $50 \%$ mujeres), que exploraron cuatro aspectos: tabaquismo, uso del telefono móvil, opinión sobre el contenido y redacción de los mensajes y opinión sobre el servicio SmokefreeTXT.

Se analizaron, tanto en los grupos focales como en las entrevistas semiestructuradas, las palabras clave (Figura 1) que permiten la retroalimentación al usuario y una muestra de 68 SMS elegidos aleatoriamente.

Posteriormente, se formaron dos grupos focales con personal de salud, el primero con expertos en el tratamiento del tabaquismo y el otro conprofesionales que atienden padecimientosderivados de este problema, quienes reflexionaron sobre las dificultades que enfrentan cotidianamente; el uso de TIC en su quehacer profesional y su opinión sobre SmokefreeTXT. Además, revisaron las posibles causas de la incomprensión de algunos SMS para su modificación y se les pidió generar otros mensajes para abordar las BCT ausentes, que fueron: Informacióndel comportamiento y experiencias de otros, Importancia de la interrupción brusca, Conservación de recursos mentales y Expectativas del programa (28)-, a partir de sus conocimientos y experiencia.

\section{Análisis \\ Las sesiones de trabajo y las entrevistas fueron grabadas con un equipo Canon-HFR700 y transcritas en Microsoft Word, analizando la información con el software ATLAS.ti 6.0, lo que permitió clasificar dominios y categorías de acuerdo a la propuesta de Colantonio et al (11) para integrar la biblioteca adaptada de mensajes SmokefreeTXT para población mexicana, particularmente veracruzana.}

\section{Resultados}

\section{Percepciones del tabaco y su abandono: Fumadores}

En los grupos focales de fumadores participaron jóvenes entre 18 y 22 años, con una edad promedio de 20; en contraste, los entrevistados tenían una edad que fluctuaba entre 25 y 60 años, con una edad promedio de 46 años (Tabla 1). Entre las razones para fumar destacaron los aspectos positivos asociados al tabaco, tales como la sensación de relajamiento, la disminución de la ansiedad y la posibilidad de lograr una mayor concentración para estudiar. En cuanto a las razones por las que desearían dejar de fumar, detectamos diferencias entre los más jóvenes y los mayores de 45 años, ya que los primeros se enfocan en el efecto que el tabaco tiene en su apariencia física, como dientes o uñas amarillentas, mientras los mayores destacaron el mal ejemplo para sus hijos, las molestias que provocan a los no fumadores y el maltrato a su propio cuerpo. 
Tabla $\mathbf{N}^{\mathbf{0}} \mathbf{1}$ : Características sociodemográficas y del uso de tecnologías de los participantes en los grupos focales.

\begin{tabular}{|c|c|c|c|}
\hline Característica & $\begin{array}{c}\text { Grupo focal } 1 \\
n=10\end{array}$ & $\begin{array}{c}\text { Grupo focal } 2 \\
n=10\end{array}$ & $\begin{array}{c}\text { Entrevistas } \\
n=12\end{array}$ \\
\hline Edad promedio & 18.3 años & 21.8 años & 46 años \\
\hline Sexo & $100 \%$ masculino & $\begin{array}{l}50 \% \text { masculino } \\
50 \% \text { femenino }\end{array}$ & $\begin{array}{l}50 \% \text { masculino } \\
50 \% \text { femenino }\end{array}$ \\
\hline Escolaridad & $\begin{array}{l}90 \% \text { estudian } \\
\text { bachillerato }\end{array}$ & $\begin{array}{l}100 \% \text { estudiantes } \\
\text { universitarios }\end{array}$ & $\begin{array}{c}8 \% \text { Sabe leer y escribir } \\
42 \% \text { Preparatoria } \\
17 \% \text { Licenciatura } \\
33 \% \text { Posgrado }\end{array}$ \\
\hline Ocupación & $\begin{array}{c}70 \% \text { trabajan de manera } \\
\text { asalariada aparte de } \\
\text { estudiar }\end{array}$ & $\begin{array}{c}70 \% \text { trabajan de manera } \\
\text { asalariada aparte de estudiar }\end{array}$ & $\begin{array}{l}75 \% \text { trabajan de manera } \\
\text { asalariada } \\
25 \% \text { trabajan por cuenta } \\
\text { propia }\end{array}$ \\
\hline $\begin{array}{l}\text { Número promedio de } \\
\text { cigarrillos que fuman } \\
\text { diariamente }\end{array}$ & 4.7 & Menos de 1 & 8 \\
\hline $\begin{array}{l}\text { Promedio de edad en la que } \\
\text { iniciaron a fumar }\end{array}$ & 12.2 años & 17.5 & 17 años \\
\hline $\begin{array}{l}\text { Tecnología de uso más } \\
\text { frecuente }\end{array}$ & $100 \%$ teléfono móvil & $\begin{array}{c}100 \% \text { teléfono móvil } \\
100 \% \text { laptop }\end{array}$ & $\begin{array}{c}100 \% \text { teléfono móvil } \\
58 \% \text { laptop }\end{array}$ \\
\hline Uso de SMS & $90 \%$ todos los días & $\begin{array}{c}30 \% \text { todos los días } \\
30 \% \text { al menos una vez a la } \\
\text { semana } \\
40 \% \text { al menos una vez al } \\
\text { mes }\end{array}$ & $100 \%$ todos los días \\
\hline
\end{tabular}

-En cuanto a la disposición para dejar de fumar, los más jóvenes asumieron dos posturas contrarias: algunos consideraban no tener un problema de adicción, debido a que no consumían diariamente, mientras que los que estaban en tratamiento por el consumo abusivo de drogas ilegales, consideraban necesitar del tabaco para eliminar la ansiedad derivada de la abstinencia de la marihuana. Por su parte, los mayores expresaron que lo habían intentado en varias ocasiones o que ya estaban en proceso de dejar de fumar; este último grupo visualizaba como dificultades para abandonar este hábito el afrontamiento del estrés, mientras que el primer grupo no contempló dificultades.

\section{Evaluación de SmokefreeTXT: Fumadores}

El análisis de la opinión sobre SmokefreeTXT, mostró que la mayoría de las opiniones fueron positivas, ya que los SMS se consideraron motivadores para apoyar el proceso que inicia cuando se decide dejar de fumar y que concluye cuando se logra hacerlo. Sin embargo, todos reconocieron que la efectividad del método está condicionada a la convicción de dejar de fumar por parte del sujeto.

Cuando se preguntó sobre la disposición para pagar por el servicio SmokefreeTXT, la respuesta se condicionó a la efectividad del método. Los más jóvenes consideraron que este tipo de servicio está dirigido a personas mayores, como sus padres quienes tendrían que vencer las dificultades que tienen ante el uso del teléfono móvil.

Respecto al contenido de los SMS, no se detectaron diferencias en las opiniones por sexo y edad; la mayoría de los participantes sugirieron modificar el trato interpersonal, utilizando el tuteo para provocar la confianza del usuario. Los participantes más jóvenes se inclinaron por mensajes con contenido desafiante y expresaron desagrado ante los de contenido fatalista, como los mostrados en las cajetillas. También se identificó la necesidad de añadir un mensaje para generar la reflexión del usuario sobre las ventajas económicas de dejar de fumar y la oportunidad de ahorrar ese recurso. 
Como resultado del proceso de análisis inicial de los SMS, se modificó la redacción de 55 mensajes (32\%) para hacerlos más comprensibles a la población mexicana; a pesar de ello, los participantes encontraron que 10 SMS no eran suficientemente claros debido al uso de ciertas palabras, como: los que incluían el vocablo "antojos", ya que en México se asocia a la comida. Tampoco resultó clara la palabra clave HUMOR, ya que a los mexicanos les sugiere que recibirán algún mensaje humorístico y no apoyo adicional del servicio, en caso de requerirlo. La palabra PARAR, como indicación para cancelar el servicio, no fue comprendida y además en la intervención se usaba de manera inconsistente, puesto que algunos mensajes utilizaban la palabra BAJA con idéntico fin, misma que fue seleccionada como más adecuada en la adaptación.

\section{Evaluación de SmokefreeTXT: Profesionales de salud}

En el Tabla N ${ }^{\circ} 2$ presentamos las características sociodemográficas y el uso que hacen de las TIC, en su labor cotidiana, los profesionales de la salud que participaron.

Tabla N²: Características sociodemográficas y del uso de tecnologías del profesionales de la salud.

\begin{tabular}{|c|c|c|}
\hline Característica & $\begin{array}{l}\text { Expertos en el tratamiento de } \\
\text { adicciones } \\
n=10\end{array}$ & $\begin{array}{c}\text { Profesionales de la salud que } \\
\text { atienden problemas relacionados con } \\
\text { el tabaquismo } \\
n=6\end{array}$ \\
\hline Edad promedio & 42.5 años & 47 años \\
\hline Sexo & $\begin{array}{l}40 \% \text { masculino } \\
60 \% \text { femenino }\end{array}$ & $\begin{array}{l}17 \% \text { masculino } \\
83 \% \text { femenino }\end{array}$ \\
\hline Tipo de tratamiento que proporcionan & $\begin{array}{l}50 \% \text { deshabituación tabáquica } \\
50 \% \text { tratamiento de otras drogas }\end{array}$ & $\begin{array}{l}33 \% \text { primer nivel de atención } \\
67 \% \text { segundo nivel de atención }\end{array}$ \\
\hline $\begin{array}{l}\text { Años promedio de experiencia } \\
\text { profesional }\end{array}$ & 19.5 años & 20 años \\
\hline Profesión & 100\% psicólogos & $\begin{array}{l}83 \% \text { médicos } \\
17 \% \text { odontólogos }\end{array}$ \\
\hline Con estudios de posgrado & $\begin{array}{l}60 \% \operatorname{con} \\
40 \% \sin \end{array}$ & $\begin{array}{l}100 \% \text { con especialidad médica u } \\
\text { odontológica }\end{array}$ \\
\hline Tecnología de uso más frecuente & $\begin{array}{l}100 \% \text { teléfono móvil } \\
50 \% \text { computadora de escritorio }\end{array}$ & $\begin{array}{l}100 \% \text { teléfono móvil } \\
100 \% \text { laptop }\end{array}$ \\
\hline Uso de SMS & $\begin{array}{l}50 \% \text { todos los días } \\
40 \% \text { al menos una vez a la } \\
\text { semana } \\
10 \% \text { al menos una vez al mes }\end{array}$ & $\begin{array}{c}50 \% \text { todos los días } \\
50 \% \text { al menos una vez a la semana }\end{array}$ \\
\hline
\end{tabular}

El grupo que integró a profesionales de la salud que proporcionan tratamiento a fumadores, identificó que el principal problema que enfrentan es la ansiedad provocada por el retiro de la sustancia. Otro elemento importante, se refiere a las creencias o mitos que los pacientes tienen sobre el tabaco, lo que explica que le otorguen un "poder divino".

Respecto a los SMS, consideraron que son un gran apoyo para el tratamiento pese al reducido número de caracteres en cada mensaje, lo que no permite profundizar en ciertos aspectos. Todos coincidieron en que el trato interpersonal debe generar confianza y que es necesario resaltar más los beneficios para la salud de dejar de fumare incluir información sobre servicios locales de deshabituación tabáquica.

El grupo focal de especialistas en enfermedades atribuibles al tabaco, refiriócomo práctica rutinaria indagar sobre consumo de tabaco en los pacientes que acuden a consulta, prioritariamente cuando presentan síntomas relacionados con el hábito. Respecto al uso de SmokefreeTXT, valoraron que se trata de una buena alternativa para dejar de fumar además de ser barata, pero señalaron que el formato es limitado y que los SMS ya casi no están siendo utilizados, debido al surgimiento de nuevas aplicaciones tecnológicas. También 
consideraron que el tuteo es más adecuado para generar un acercamiento personalizado y mayor confianza.

\section{Integración de la biblioteca de mensajes de texto adaptada}

Las reflexiones realizadas por el equipo investigador, los grupos focales y los entrevistados, permitió identificar los distintos dominios, elaborar nuevos mensajes y precisar otros. En cuanto a los dominios se identificaron tres y 13 categorías de análisis (figura $\mathrm{N}^{\circ} 2$ ). El primer dominio "Contenido del mensaje", incluyó cuatro categorías: mitos, vacíos teóricos, servicios locales y otras ventajas de la deshabituación tabáquica.

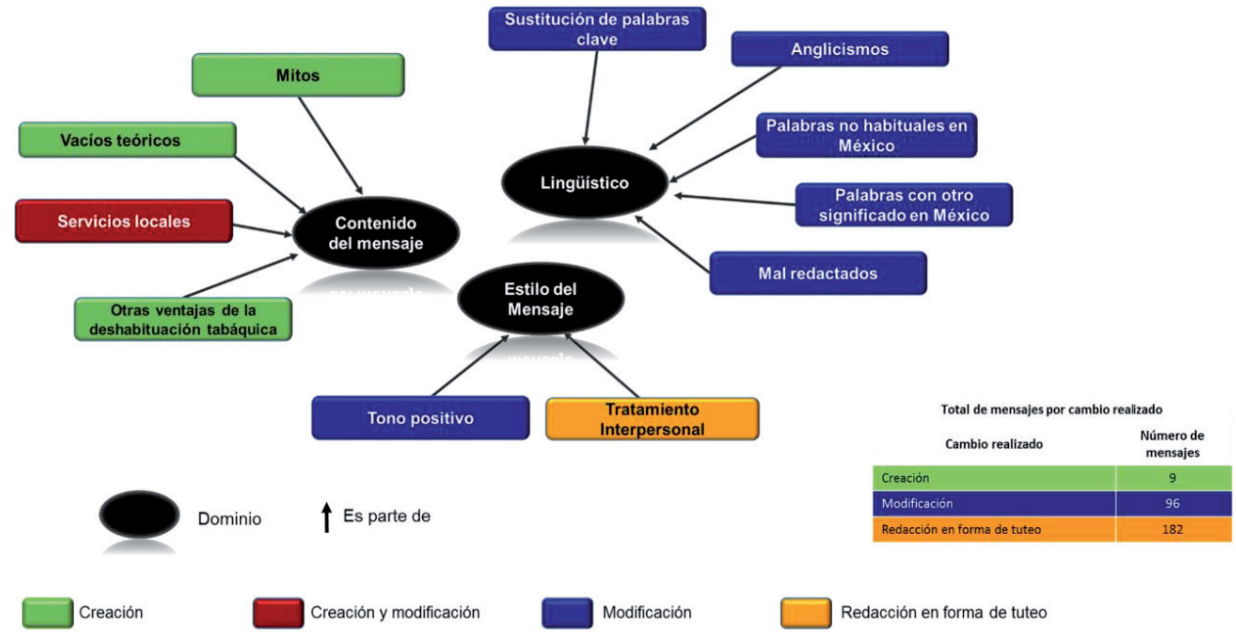

Figura N²: Mapa mental de los dominios y categorías identificados durante el proceso de adaptación de los mensajes de SmokefreeTXT en México

En la categoría mitos, se crearon tres nuevos SMS que abordan la creencia de que el tabaco ayuda a afrontar problemas personales; aumenta el rendimiento intelectual y no afecta a la salud, si se hace esporádicamente. En la segunda categoría, se crearon cuatro SMS para generar las BCT que los expertos consideraron ausentes y necesarias para el contexto mexicano y veracruzano. En la categoría servicios locales, se creó un nuevo SMS y se modificaron cuatro que brindan información sobre servicios de deshabituación tabáquica en Xalapa. En la última categoría, se generó un mensaje que apela a ahorrar dinero en lugar de gastarlo en cigarrillos.

En el segundo dominio "Estilo del mensaje", se identificaron las categorías tratamiento interpersonal y tono positivo, se modificaron todos los SMS para utilizar el tuteo y enfatizar los mensajes positivos más que los catastróficos.

En el último conjunto, "Dominio lingüístico", se ubicaron a las categorías relacionadas con aspectos gramaticales: Sustitución de palabras clave, Anglicismos, Palabras no habituales en México, Palabras con otro significado en México y Mal redactados, lo que llevó a cambiar el vocablo PARAR por BAJA, para indicar que el participante desea cancelar el servicio, en 11 SMS; a sustituir palabras de origen inglés por sus equivalentes en español en tres SMS (chance por oportunidad)y a modificar palabras que no fueron comprendidas debido a que no son usuales (como hito por éxito) en cinco SMS. Otros 16 SMS se modificaron dentro de la categoría Palabras con otro significado en México, ya que se interpretaron de manera ambigua (como antojo por deseo) y finalmente, se cambiaron 55 SMS dentro de la última categoría.

Como resultado de todo el proceso, de los 173 SMS originales de SmokefreeTXT, se modificaron 96 y se crearon nueve, con lo cual la biblioteca de este servicio para población mexicana quedó integrada por $182 \mathrm{SMS}$. 
Las modificaciones a la redacción de algunos SMS se hicieron buscando una mejor comprensión por los hablantes de español en México y particularmente en Veracruz así como eliminar los anglicismos, lo que coincide con las de modificaciones realizadas en la adaptación de STOMP a Reino Unido (12).

Tres de los dominios identificados coinciden con los detectados por Colantonio (11) aunque la nomenclatura es distinta ya que al dominio, identificado por estos autores como expresiones y palabras, fue nombrado dominio lingüístico en este estudio, debido a que no sólo se consideraron categorías como "anglicismos" o "palabras con otro significado en nuestro país", sino que también modificamos aquellos SMS con errores tipográficos, faltas de ortografía o redacción confusa.

Se encontró que la opinión sobre SmokefreeTXT fue positiva, aunque los participantes sólo conocieron la descripción del servicio,lo que coincide con los resultados del estudio realizado por Andrews en fumadores mexicanos que manifestaron interés en utilizar un servicio similar cuando les fue descrito (29); también concuerda con Colantonio (11) ya que los expertos señalaron que este tipo de intervenciones representa un gran apoyo para el tratamiento de la deshabituación tabáquica. Los resultados del mismo estudio coinciden con los nuestros en cuanto a que también realizaron modificaciones derivadas del tratamiento interpersonal para generar confianza en los participantes, en el caso de México se optó por el tuteo mientras en Argentina por la opción "vos" (11).

Con relación a la percepción de que el estilo de algunos SMS de SmokefreeTXT eran fatalistas, similares a los mensajes del empaquetado de los cigarros, coincide con lo encontrado por Ybarra y colaboradores, quienes sugieren que los adultos jóvenes prefieren SMS positivos (30); en contraste, difieren de lo reportado por Colantonio y colaboradores (11), en donde los participantes se inclinaron por mensajes con información sobre efectos negativos del tabaquismo, lo que sus autores atribuyen a que en Argentina se obligó a los fabricantes a imprimir mensajes sobre las consecuencias adversas del tabaquismo en las cajetillas de cigarros a partir de 2011 mientras que en México se observa desde 2004 (31). Otra coincidencia con el estudio de Argentina fue que los participantes solicitaron que al usar la palabra "antojos" en los SMS, se especificara que era de fumar, ya que en ambos países, el uso de esta palabra se relaciona con antojos de comida en general y particularmente durante el embarazo (11).

La mayor parte de los países de América Latina han suscrito el CMCT y algunos, como Uruguay, se han distinguido por su liderazgo en la aplicación de ciertas estrategias de este convenio $(8,1)$; sin embargo, una de las áreas en donde se registra menor avance se refiere al cumplimiento de los compromisos del artículo 14 (32), por lo cual la implementación de servicios de envió de SMS a gran escala podrían constituir una alternativa de bajo costo, ya que pese a la disposición de los participantes de este estudio de pagar, las condiciones socioeconómicas de la mayor parte de esta población hacen difícil el pago de servicios onerosos.

\section{Conclusión}

Aunque el proceso de adaptación transcultural concluye una vez que se ha probado su eficacia en la población destino, podemos asentar que la intervención se enriqueció con la precisión y generación de nuevos mensajes y que se amplió el conocimiento de las variantes culturales. Una de las riquezas de este trabajo descansa en sus participantes, ya que se logró que los fumadores fueran representantes de una tipología amplia entre los que se encuentran grupos de hombres, mujeres, jóvenes, adultos, estudiantes universita-

${ }^{4}$ Actualmente se realiza un estudio piloto para probar la eficacia de SmokefreeTXT en el estado de Veracruz. 
rios y los que consumen otras drogas. Asimismo, participaron profesionales de la salud, expertos en el tratamiento del tabaquismo y en las enfermedades asociadas. Sin embargo y reconociendo que esta fue una limitación del trabajo, recomendamos a otros investigadores interesados en realizar adaptaciones transculturales de este tipo, que integren a un mayor número de fumadores y de grupos focales para lograr la saturación del discurso en el análisis del total de SMS de cada servicio.

Aunque México comparte algunas características con países latinoamericanos, cada nación tiene su propia identidad cultural y lingüística, lo que sustenta la necesidad de realizar adaptaciones transculturales que, en adelante, podrán usar el método propuesto en este trabajo. Posteriormente, al probarse la eficacia y aceptación de la intervención en una muestra representativa de la población blanco, nuestra biblioteca podrá ser utilizada como referente para las adaptaciones transculturales para contribuir a atender este problema en la región.

\section{Bibliografía}

1. Organización Panamericana de la Salud. Informe sobre Control del Tabaco para la Región de las Américas. Washington, DC: OPS; 2013. 1-66 p.

2. Guerrero-López CM, Muños-Hernández JA, Sáenz de Miera-Juárez B, ReynalesShigematsu LM. Consumo de tabaco, mortalidad y política fiscal en México. Salud Publica Mex. 2013;55(Suppl 2):S276-81.

3. Organización Mundial de la Salud. Directrices para la aplicación del artículo 14 del Convenio Marco de la OMS para el Control del Tabaco. 2010.

4. Whittaker R, McRobbie H, Bullen C, Borland R, Rodgers A, Gu Y. Mobile phonebased interventions for smoking cessation. Cochrane Database Syst Rev. 2012;(11).

5. Ghorai K, Akter S, Khatun F, Ray P. mHealth for smoking cessation programs: A systematic review. J Pers Med. 2014;4(3):412-23.

6. Guerriero C, Cairns J, Roberts I, Rodgers A, Whittaker R, Free C. The cost-effectiveness of smoking cessation support delivered by mobile phone text messaging: Txt2stop. Eur J Heal Econ. 2013 Oct;14:789-97.

7. Ruiz Pacheco M, Álvila de Tomás JF, Sánchez-Rodrigo JV, Bravo, Minué Lorenzo C, López Bravo R. El uso de las nuevas tecnologías en el tratamiento del tabaquismo. En: Jiménez-Ruiz CA, Fagerström K, eds. Tratado de tabaquismo. $3^{\circ}$ ed. Madrid, España: Auka Médica; 2011.

8. Raw M, Mackay J, Reddy S. Time to take tobacco dependence treatment seriously. Lancet. Elsevier Ltd; 2016;387(10017):412-3.

9. United Nations Development Programme. Mobile Technologies and Empowerment: Enhancing human development through participation and innovation [Internet]. New York; 2012. Hallado en: http://www.undp.org/content/undp/en/home/ librarypage/democratic-governance/access_to_informationande-governance/mobiletechnologiesprimer.html. Acceso el 16 de abril de 2013.

10. Free C, Knight R, Robertson S, Whittaker R, Edwards P, Zhou W, et al. Smoking cessation support delivered via mobile phone text messaging (txt2stop): a singleblind, randomised trial. Lancet. Elsevier Ltd; 2011 Jul 2;378(9785):49-55.

11. Colantonio LD, Pena L, Whittaker R, Mejia RM. Cross-cultural adaptation of a text message-based program for smoking cessation in Buenos Aires, Argentina. Nicotine Tob Res. 2015;1-7.

12. Free C. Developing and Adapting a Text Messaging Intervention for Smoking Cessation from New Zealand for the United Kingdom. En: J. Donner and P. Mechael, editor. mHealth in Practice: Mobile technology for Health Promotion in the Developing World. London, UK: Bloomsbury; 2012. p. 46-61.

13. Abroms L. Mobile Phones \& Smoking Cessation. En: The Future of Tobacco Control: Implications for the Prevention of Youth Tobacco Use in Virginia Conference. 
2015. p. 1-43.

14. Lichtenstein E, Zhu S-H, Tedeschi G. Smoking Cessation Quitlines: An Underrecognized Intervention Success Story. Am Psycol. 2010;65(4):252-61.

15. Businelle MS. The potential of mHealth for tobacco dependence treatment: Domestic and international examples from NCI'S smokefree.gov initiative. Nicotine Tob Res. 2014;16(7):1.

16. Augustson E. The National Cancer Institute (NCI) SmokefreeTXT (Text Message) Program Evaluation. 2013.

17. Okuyemi KS, Nollen NL, Ahluwalia JS. Interventions to facilitate smoking cessation. Am Fam Physician. 2006 Jul 15;74(2):262-71.

18. Whittaker R. Panel on Adapting MHealth Interventions for New Contexts \& Countries. En: Medicine 20 World Congresse on Social Media, Mobile Apps, and Internet/Web 20. 2014.

19. Barrera M, Castro F, Strycker L, Toobert D. Cultural Adaptations of Behavioral Health Interventions: A Progress Report. J Consult Clin Psychol. 2013;81(2):196205.

20. Sepulveda R, Molina T, Molina R, Martínez V, Gonzalez E, George M, et al. Adaptación transcultural y validación de un instrumento de calidad de vida relacionada con la salud en adolescentes chilenos. Rev Med Chile. 2013;141:1283-92.

21. Michie S, Richardson M, Johnston M, Abraham C, Francis J, Hardeman W, et al. The behavior change technique taxonomy (v1) of 93 hierarchically clustered techniques: Building an international consensus for the reporting of behavior change interventions. Ann Behav Med. 2013;46(1):81-95.

22. Prochaska J, DiClemente C. Stages and processes of self-change of smoking: toward an integrative model of change. J Consult Clin Psychol. 1983;51(3):390-5.

23. Bandura A. Social cognitive theory: An Agentic Perspective. Annu Rev Psychol. 2001;52:1-26.

24. Fishbein M, Ajzen I. Belief, Attitude, Intention, and Behavior: An Introduction to Theory and Research. Reading, MA: Addison-Wesley; 1975.

25. West R. The PRIME Theory of motivation as a possible foundation for addiction treatment. En: Henningfield P, Santora, West R, eds. Drug Addiction Treatment in the 21st Century: Science and Policy Issues. Baltimore: John's Hopkins University Press; 2007.

26. Cole-Lewis H. Behavior Change Techniques used by the Teen SmokefreeTxt Intervention. 2011.

27. Michie S, Free C, West R. Characterising the "Txt2Stop"Smoking Cessation Text Messaging Intervention in Terms of Behaviour Change Techniques. J Smok Cessat. 2012; 7:55-60.

28. Andrews L, Cacho-Elizondo S, Drennan J, Tossan V. Consumer acceptance of an SMS-assisted smoking cessation intervention: a multicountry study. Heal Mark Q. 2013 Jan;30:47-62.

29. Ybarra ML, Prescott TL, Holtrop JS. Steps in Tailoring a Text Messaging-Based Smoking Cessation Program for Young Adults. J Heal Commun. 2014;1-15.

30. Thrasher JF, Pérez-Hernández R, Arillo-Santillán E, Barrientos-Gutiérrez I. Hacia el consumo informado de tabaco en México: Efecto de las advertencias con pictogramas en población fumadora. Salud Publica Mex. 2012;54(3):242-53.

31. Sandoval RC, Blanco A. Estado de la implementación del Convenio Marco para el Control del Tabaco en la región de las Américas. Salud Publica Mex. 2010;52(SUPPL.2).

32. Free C, Phillips G, Galli L, Watson L, Felix L, Edwards P, et al. The effectiveness of mobile-health technology-based health behaviour change or disease management interventions for health care consumers: a systematic review. PLoS Med. 2013 Jan;10(1):1-46. 